\title{
Naturalismo biológico
}

\author{
CARLOS CASTRODEZA*
}

I. ¿POR QUÉ NATURALISMO BIOLÓGICO?

EN CIERTO MODO UNA REFERENCIA AL NATURALISMO BIOLÓGICO puede parecer redundante. Porque si el naturalismo es el tratamiento de los problemas filosóficos desde la ciencia, la perspectiva biológica de todo problema filosófico es algo evidente. Sin embargo, es importante añadir ese predicado a modo de énfasis por dos razones.

La primera es que la referencia naturalista normalmente ha provenido de la psicología cognitiva ${ }^{1}, y$ últimamente de la sociología ${ }^{2}$, y tanto psicólogos como sociólogos parecen tener, a la vista de las publicaciones al respecto, una concepción de las ideas biológicas -que, es de suponer, dan por sentadas- un tanto esencialistas 3 .

La segunda es que cuando existe una alusión directa a la biología, es a un darwinismo más bien retórico y siempre acrítico. Es decir, se da por buena la teoría de Darwin más o menos actualizada, sin tener en cuenta cómo pueden cambiar el panorama naturalista las disensiones existentes, de darse al traste un día con la ortodoxia darwiniana vigente.

* Universidad Complutense de Madrid.

1 Véase el locus classicus, Epistemology Naturalized en W. V. O. Quine 1969, y, por ejemplo, P. M. Churchland 1984, o R. Giere 1988.

2 La referencia es a la obra de los sociólogos del conocimiento en general; véase, especialmente, A. V. Cicourel 1974, desde la perspectiva más propiamente cognitiva, o D. Hull 1988, desde el enfoque de la sociología de la ciencia.

3 D. Hull, 1988 es la excepción. 
En fecha reciente, Alex Rosenberg, filósofo, que fue, de la ciencia económica, y hoy filósofo de la biología, ha publicado una 'guía de campo' del naturalismo actual ${ }^{4}$, en el que además de críticas controvertidas -en el sentido ya apuntado-- existen omisiones significativas que exijen las debidas puntualizaciones. En cualquier caso, el escrito de Rosenberg es importante porque, cuando menos, suministra un buen hilo conductor de las cuestiones pertinentes.

Oficialmente, puede atribuirse a E. Nagel, el origen de la cuestión naturalista en filosofía en una alusión que hiciera en $1955^{5}$. Nagel venía a considerar que la problemática filosófica no existe y que sólo la tesitura científica tiene sentido. Esta actitud, claro está, es una derivación lógico positivista sin más. Como es bien sabido, para este movimiento no hay más filosofía que la Lógica y ésta no sería más que un formalismo para la expresión de los problemas científicos -únicos problemas genuinos (los demás problemas serían reducibles a los científicos, precisamente con la ayuda de esa lógica que facilitaría la tarea al respecto).

Asimismo, la crítica tradicional de que el naturalismo no suministra una garantía independiente para la actividad epistemológica, queda rechazada por Nagel por medio de un argumento típicamente positivista (en una acepción instrumentalista del término): nunca hay premisa inicial evidente alguna, siempre hay que retrotraerse para caer en la regresión infinita de costumbre, por lo que la única garantía epistémica es la evidencia que ha servido para obtener conocimiento en el que podemos confiar, es decir, el conocimiento que nos haya sido útil6. El problema que surge de inmediato es que conocimiento útil es todo. El usuario del conocimiento que sea es quién le da la dimensión pragmática que proceda, como, por ejemplo, dejó bien claro en su día William James en esos casos extremos que relata en su Variaciones de la Experiencia Religiosa. En definitiva, paradójicamente, por este camino el naturalismo nageliano nos conduciría a un 'todo vale' en la dimensión más feyerabendiana del término.

Pero por si esta dificultad no fuera suficiente, existe otro problema derivado del primer naturalismo de impacto en el mundo de la filosofía,

4 A. Rosenberg 1996.

5 Nagel loc. cit. en A. Rosenberg, op. cit., pp. 1-4.

6 La expresión original es Reliable Knowledge. Por supuesto que esta crítica sí que es un auténtico ácido universal (ver más adelante), porque no hay epistemología que trascienda la trampa de la autorreferencia. 
que fue el de Quine, no ya el que explicitara en 19697 -como naturalismo cognitivo (psicología cognitiva) - sino el naturalismo implícito en su famoso escrito de 1951, Dos Dogmas del Empirismo ${ }^{8}$, porque si la diferencia, de que ahí se trata, entre lo analítico y lo sintético no es nítida, la misma lógica formal queda en entredicho9: el último refugio de la filosofía desaparece y se desemboca, aparentemente sin remedio, en los supuestos excesos epistemológicos de la sociología del conocimiento de nuestros días en que todo -incluida, claro está, la lógica formal-sería una construcción social sin más, aunque se le pueda dar una dimensión utilitarista.

¿Y dónde nos conduciría, como situación límite, el 'todo vale' en un sentido amplio, junto a la supuesta construcción social de la realidad en su totalidad? Dejando de momento a un lado la cuestión de que esa larga pregunta necesita una detallada matización, la referencia final sería a la existencia de una estrategia de supervivencia más o menos funcional. Para muchos se entra en el mundo de Darwin, es decir-siguiendo la tradición de P. L. Berger y Th. Luckmann $10 \ldots$ en el universo simbólico de la selección natural, o sea el 'todo vale' -darwiniano esta vez- o el ácido universal que todo lo corroe, como lo denomina Dennett 11 .

\section{LA APROXIMACIÓN BIOLÓGICA SUPERFICIAL}

\section{LA 'SELECCIÓN NATURAL’ COMO SUPUESTO TEÓRICO}

Pero el mundo de Darwin es simplemente un mundo biológico posible. Existen otros mundos biológicos posibles en los que la selección natural tiene un papel secundario 12 (dejando aparte aquéllos otros mundos caracterizados por un finalismo teleológico que serían antinaturalistas por definición).

Es más, las dos razones que aboga Rosenberg para justificar la adopción del darwinismo ortodoxo13 por los naturalistas de última generación son cuestionables. La primera justificación sería que, al parecer, en

7 W. V. O. Quine op. cit.

8 W. V. O. Quine 1951.

9 Aunque ya quedara casi plagada de incertidumbres después de Gödel.

10 La referencia es a su obra clásica de 1966 avalada biológicamente por K. Lorenz 1977, pp. 196, 220, 290.

11 D. Dennett 1995, aunque éste no le daría la dimensión antiepistémica que aquí se trasluce.

12 Véase C. Castrodeza 1997.

13 Existen heterodoxias darwinianas que darían lugar a naturalismos distintos como se puede constatar ampliamente en Hull op. cit. 
el mundo darwiniano se resuelve el problema teleológico, o de las causas finales -solución que los biólogos denominan desde hace más de tres décadas teleonomía 14 . Pero en realidad, este problema se resolvió ya cuando se temporalizó, secularizándose, la gran cadena del ser ${ }^{15}$ en la Ilustración (sobre todo en la Ilustración Francesa, más que en la escocesa, la inglesa o la alemana). Lo cierto es que hubo temporalizaciones secularizadas (naturalistas) diferentes que han dado lugar a los mundos biológicos distintos a que se ha hecho mención.

Impugnar, que no refutar, la segunda razón de Rosenberg es mucho más interesante, aunque la base argumental sea la misma. La razón que propugna Rosenberg es que si existe una teoría bien establecida que nos pueda enseñar algo acerca de nosotros mismos es la de Darwin. El filósofo americano se refiere sin duda a la explicación del comportamiento humano que se predica de la metáfora del gen egoísta ${ }^{16}$. Pero como muy bien observa Matt Ridley17, el comportamiento egoísta del gen (en realidad del replicador 18 ), no implica un comportamiento egoísta al nivel individual. Ahora bien, si lo que tenemos entre manos es un comportamiento altruista puede ser, o bien porque sea genuinamente altruista, o bien porque, por el contrario, se remita a la conveniencia egoísta de los replicadores subyacentes. Experimentalmente, seguramente es imposible dirimir esa disyuntiva. De hecho, Williams en su famoso tratado de 1966, afirma que la reducción del nivel de selección individual -o supraindividual-al nivel de selección genético no es más que una aplicación de la navaja de Occam 19 .

Lo que sucede es que si acudimos a otros modelos evolutivos -naturalistas, pero no darwinianos- la selección natural no es algo destacable. Sí es destacable si es algo que se instrumenta en gran medida en el proceso evolutivo (en el darwinismo), pero no si esa instrumentación se hace en menor medida en las alternativas existentes (neutralismo ${ }^{20} \mathrm{y}$

14 Así la teleonomía sería a la teleología lo que la astronomía a la astrología.

15 Véase la obra clásica de A. O. Lovejoy 1936.

16 Metáfora introducida 'oficialmente' por R. Dawkins en 1976, con los importantes precedentes de W. Hamilton 1964, M. Ghiselin 1974, E. O. Wilson 1975 y, sobre todo, G. C. Williams 1966.

17 Véase M. Ridley 1996.

18 Para entender esta importante diferencia, que hace obsoleto el concepto de gen, véase de nuevo D. Hull op. cit.

19 Es más, la ortodoxia darwiniana exige lógicamente esa aplicación de la navaja de Occam, como se constata fácilmente en la obra de R. Dawkins op. cit. y que G. C. Williams op. cit. menciona explícitamente.

20 M. Kimura 1983. 
direccionalismo no teleológico ${ }^{21}$ ). De otra manera, la selección natural, no la teoría, es algo evidente que en la teoría de Darwin tiene una relevancia evolutiva, que no en las otras teorías.

\section{2. 'LA SELECCIÓN NATURAL' COMO MODELO GENERAL}

Pero lo que es más relevante, sin acudir a heterodoxias (ni mucho menos a teorías alternativas), es que el modelo que plantea Rosenberg al respecto 22 es, cuando menos, equívoco.

$\mathrm{Su}$ primera categorización es correcta, pero es que realmente es definitoria. En efecto, tomar como base epistemológica cualquier tipo de consideración filosófica al margen de la ciencia sería antinaturalista. La segunda tesitura también es aceptable (y, de hecho, está contenida en la caracterización anterior), es decir, se impone el cientismo -sobre todo a partir de la ciencia más asentada- que será la base para cualquier consideración epistemológica o metafísica. Pero el tercer supuesto ya es controvertido, en cuanto que se considera la teoría darwiniana como, en gran medida, el fundamento naturalista más pertinente en lo que se refiere a su conexión con la condición humana. Como se viene diciendo, no es que haya ya heterodoxias darwinianas relativamente bien establecidas, sino que existen otras corrientes importantes en las que la selección natural no tiene protagonismo evolutivo alguno.

La cuarta dimensión, referente al modelo naturalista de Rosenberg, ya no es admisible. Según dicho autor, el naturalismo abogaría por la racionalidad, carácter acumulativo y progreso de la ciencia. Y esto no es aceptable desde el mismo modelo darwiniano ortodoxo, ya que si el proceso de selección natural siempre es relativo al medio, si éste cambia, el proceso evolutivo es errático y/o reversible (siempre siguiendo la ley de Dollo, claro está), y si el medio no cambia la selección natural estimula la especialización orgánica al precio de una mayor vulnerabilidad, caso de que el medio cambie alguna vez.

Rotundamente, si una argumentación a favor del progreso biológico no tiene cabida en la teoría de Darwin ${ }^{23}$, tampoco la tiene a favor del progreso científico en el naturalismo darwinista, simplemente por ser éste una característica orgánica más, aunque sea adscribible al hombre (porque, asimismo desde el naturalismo, la estrategia adaptativa humana sería 1996.
B. Goodwin (1995) o S. Kauffman 1995.

A. Rosenberg op. cit., pp. 4-5.

Véase C. Castrodeza 1988 o, desde otro ángulo complementario, S. J. Gould 
una más, por mucho que se distinguiera por una racionalidad consciente que estaría ausente, en buena medida, en los demás seres vivos 24 ). Lo mismo ocurre, a este respecto claro está, en la distintas heterodoxias darwinianas.

Incidentalmente, tampoco el progreso en general tiene sitio en las alternativas a la selección natural. En el direccionalismo, aunque en principio exista un proceso de complejidad, complejidad y progreso son fenómenos independientes, aunque se puedan compatibilizar. Y en el neutralismo, ahí sí que por definición el progreso brilla por su ausencia.

En definitiva, en un naturalismo genuino toda perspectiva axiológica está de más, a no ser que se considerara como una manifestación orgánica más del organismo humano, lo que igualmente le privaría de cualquier trascendencia posible.

\section{REALISMO, ANTIRREALISMO E INSTRUMENTALISMO}

\section{LA EXTENSIÓN DEL 'FENOTIPO AMPLIADO'}

Análogamente, desde el naturalismo biológico es tan absurdo considerar que existe un mundo Real (Verdadero), como que nuestros modelos -desde una perspectiva semanticista- no tienen, en principio, nada que ver con la Realidad. Es más, desde la perspectiva que aquí se adopta, el concepto de Realidad es obsoleto, no significa nada. Abundando en la conceptualización de Dawkins, sólo seríamos conscientes de nuestro propio fenotipo ampliado 25 , que dependiendo de nuestro genoma en su interacción con el soma, se percibiría de un modo u otro (el daltónico paradigmático, por ejemplo, no percibe los colores, es decir que su fenotipo ampliado no está coloreado). De este modo, toda 'Realidad' estaría compuesta de los fenotipos ampliados de los organismos que se solapan entre sí. Dicho de un modo quizá pedante, y sólo a título ilustrativo -pero ¿en harmonía con la física vigente?-, toda percepción orgánica colapsa la función de ondas provocando, por ejemplo, que el famoso gato de Schrödinger aparezca vivo o muerto, pero si no hay percepción, la 'Realidad' es un gato que está al mismo tiempo vivo y muerto, es decir, un absurdo.

24 Racionalidad biológica, que no lógica, como confunde S. Sutherland 1996 en su, por otra parte, interesante ensayo, pero que viene precisada de un modo magistral en B. Skyrms 1996.

25 R. Dawkins 1980, o empleando una metáfora marxiana muy a propósito: 'todo' pertencce al cuerpo 'inorgánico’ del hombre (véase K. M. Stokes 1992). 
Sí existe un sentido en que los enunciados pueden ser falsos o verdaderos (con minúsculas) como ocurre cuando, por ejemplo, probamos un fármaco en un animal experimental para ver si tiene un efecto buscado o no, con respecto a un control, por lo que se confirma la hipótesis establecida o la hipótesis nula. O sea que según sean los resultados estadísticos decidimos con mayor o menor 'fuerza significativa' -nivel de significatividad-que el fármaco tiene efecto o no, es decir que el efecto es verdadero o falso. Pero esto no es más que la 'técnica' biológica de supervivencia de 'ensayo y error' instrumentada por los seres humanos en lo que podemos definir como inducción eliminativa en el lenguaje de Bacon $\rightarrow$ de Mill o, incluso, de Popper- o enumerativa en el de, por ejemplo, Reichenbach ${ }^{26}$. Sería también lo que Kitcher denomina verdad significativa ${ }^{27}$ (pero desprovista de cualquier supuesta conexión con la 'Realidad').

Y lo justamente señalado delimita el naturalismo en su desarrollo propiamente biológico. O sea que cuando Rosenberg sugiere que el naturalismo requiere una concepción realista no puede estar peor encaminado. Concebir una Realidad, ajena a toda experiencia biológica, es moverse en un vacío experiencial. Es más, desde la dirección que marca el naturalismo biológico tiene tan poca enjundia considerar la posible existencia-cognoscible o no-de una realidad independiente, como proponer que la realidad se construye socialmente, como la sociología del conocimiento científico más radical sugiere 28 . De esta manera, el relativismo como actitud epistemológica queda fuera de lugar, no así el relativismo como proceso de supervivencia en un medio específico.

La única realidad sería aquélla -se insiste, con minúscula-que afecta al individuo porque sería parte de él mismo. Esa realidad-asimismo se subraya- no está ni dada ni construida, sino simplemente debe su existencia, un tanto etérea si así se estima, al organismo u organismos de la misma 'especie'29 entre los que el solape fenotípico es lo suficientemente amplio como para que exista, según los casos, una comunicación relativamente inteligible.

Cuando cambiamos de especie, el fenotipo ampliado de la misma sólo nos es accesible antropomorfizándolo -acceso que por otra parte tendría un significado adaptativo ${ }^{30}$ - y no podemos aproximarnos de otra

26 Véase B. Gower 1997.

27 P. Kitcher 1993.

28 Véase, por ejemplo, S. Woolgar 1991.

29 La razón del entrecomillado es porque se quiere evitar todo malentendido esencialista.

30 Véase M. Douglas 1990. 
manera porque para nosotros, desde el punto de vista implicado, todo es parte de nuestro fenotipo 31 . Mientras mayor sea la distancia genética más ficticia será la posible comunicación interespecífica. Incluso dentro de la misma especie, la nuestra sin ir más lejos, se dan situaciones de incomunicación manifiesta debido a la inconmensurabilidad que pueda existir al menos entre ciertos paradigmas (en el sentido kuhniano más coloquial): léase fenotipos ampliados. A la luz de esta última apreciación, incluso la percepción del fenotipo ampliado puede cambiar en lo que sería una conversión paradigmática al uso.

En cierto modo, el único recurso a la Realidad sería el cartesiano del cogito, pero como Ayer mostró impecablemente hace ya algún tiempo ${ }^{32}$, ese recurso argumental está vacío de contenido.

Georg Lakoff expresa el mismo tipo de ideas desde sus apreciaciones a partir de la lingüística cognitiva: «Según la opinión tradicional, lo que es real es independiente del propio conocimiento. Pero si estamos en lo cierto, entonces lo que consideramos como real depende muy mucho de nuestros sistemas conceptuales. Esto es, cumplimentamos dichos sistemas conceptuales utilizando nuestros cuerpos y cerebros, y luego consideramos que esos sistemas caracterizan lo que es real [...] La idea de que nuestras categorías mentales reflejan el mundo, de que se acoplan a lo que está ahí fuera, resulta ser falsa»33. Para expresarse con más propiedad, lo que es importante en este contexto, en vez de escribir que la idea que se comunica 'resulta ser falsa', Lakoff debería haber afirmado que 'resulta ser absurda' (contradictoria).

\section{2. ÉXITO TECNOCIENTÍFICO Y REALISMO EPISTEMOLÓGICO}

Los partidarios del realismo ontológico $-\mathrm{o}$, al menos, epistemológicojustifican su actitud en el éxito instrumentalista de la ciencia ${ }^{34}$. Pero, en primer lugar, desde la visión naturalista, el éxito instrumentalista en general equivale a un traslado (en el tiempo y/o en el espacio) a un lugar mejor para la propia supervivencia. Del mismo modo, cualquier organismo se traslada a la parte del medio donde sobrevive mejor -es decir, explota la parte de su fenotipo ampliado más idónea para su supervivencia- bien de

31 T. Nagel 1974.

32 A. J. Ayer (1971).

33 G. Lakoff 1995 pp. 126-7. Véanse también las recientes consideraciones al respecto de D. Papineau 1996, capítulo introductorio.

34 Los ejemplos al respecto son incontables: D. Hull op. cit., R. Giere op. cit., P. Kitcher op. cit., C. A. Hooker 1996, etc. 
un modo pasivo, como ocurre en los seres vivos sésiles, o bien activamente, como sucede en los seres vivos con comportamiento. Trivialmente, cada organismo realiza esa operación de acuerdo con el bagaje adaptativo con el que está dotado y, por supuesto, existen estrategias que tienen más éxito que otras, estrategias que si sólo tienen operatividad a corto plazo no tienen significatividad evolutiva (teorías alternativas a la darwiniana), al contrario de si esa funcionalidad fuera a largo plazo (teoría de la selección natural). En todos los casos los organismos reorganizan su 'realidad', esto es, su propio fenotipo ampliado.

Y, en segundo lugar, el éxito en cuestión hay que analizarlo en todos sus aspectos, y no únicamente en su perspectiva más triunfalista. Dicho éxito puede muy bien significar 'pan para hoy, hambre para mañana'. No es ningún secreto que, por ejemplo, en el caso del Homo sapiens la revolución neolítica supuso, allí donde ocurrió, generalmente un deterioro del 'medio' irreversible, por no hablar ya de las secuelas, hasta la fecha, de la revolución industrial. Desde la revolución neolítica la población de seres humanos sobre la Tierra se ha multiplicado por más de mil, y existen serias dudas sobre si el hombre va a poder remontar ese deterioro creciente de su medio propiciado por el tan cacareado éxito instrumentalista 35 .

De manera que el éxito en cuestión como criterio de realismo progresista no sirve en general. Y en el caso de que, en efecto, éxito hubiera, su interpretación no favorecería decisivamente en ningún momento la postura realista al respecto. Ese éxito sería provisional. Es decir, con un éxito instrumentalista creciente o acumulado -que se traduce siempre en un éxito adaptativo-- lo que se consigue es una dependencia biológica también creciente por parte del organismo de que se trate. La situación es análoga a la de un ser vivo que parasita a otro. Llega un momento en que el parásito, en un principio facultativo, se transforma en obligado, y en una situación extrema el párasito se reduce únicamente a sus gónadas a fin de reproducirse, porque todo lo demás lo hace el huesped por él. Es como cuando la larva móvil de la ascidia al transformarse en un organismo adulto sésil, 'digiere' parte de su propio sistema nervioso que ya no necesita.

El éxito teórico es todavía más controvertido. Por ejemplo, cuando Kitcher en su obra clave, ya citada, El Avance de la Ciencia, arguye a favor de un progreso teórico en episodios como las revoluciones

35 Véanse, por ejemplo, R. Passet 1979, C. Ponting 1992, K. M. Stokes 1992 o D. Arnold 1996. 
copernicana, química de Lavoisier, o darwinista, pongamos por caso, su argumento principal es que la 'racionalidad' de los 'ganadores' se hallaba, en general, menos condicionada que la de los 'perdedores'. Es decir que no es que estos últimos fueran menos racionales que los primeros, sino que por sus circunstancias particulares se encontraban en situaciones más desfavorables para decantarse por la teoría 'mejor'. Pero aún en la tesitura muy cuestionable -sustentada a partir de los repasos 'rápidos' de Kitcher de las situaciones históricas respectivas- de que ése fuera el caso, sólo se podría argüir -siguiendo al Kuhn coloquial de siempre (no se necesita otro) - a favor de una mayor coherencia de las interpretaciones teóricas y no, en ningún momento, a favor de un mayor realismo de las interpretaciones sucesivamente ofrecidas.

\section{EL NATURALISMO EPISTEMOLÓGICO COMO CONTRADICCIÓN}

También es interesante matizar la postura de Larry Laudan que de una manera, al menos oficiosa, sería uno de los representantes más destacados de la postura instrumentalista en filosofía de la ciencia, que además se confiesa antirrealista. En efecto, Laudan estaría en profundo desacuerdo con Kitcher en lo que respecta al éxito teórico -en parte por las razones que aquí se esgrimen- pero no así en lo que se refiere al éxito instrumentalista 36 (aunque aquí Laudan tiene problemas con los sociólogos del conocimiento más radicales al mantener éstos que los éxitos de Laudan no son más que construcciones sociales).

Cuando Rosenberg le critica a Laudan por su antirrealismo dice que si éste «tiene razón, entonces el naturalismo no requiere realismo. Pero privado del propósito de llegar a la verdad que tiene toda teoría, el naturalismo requiere una plataforma epistémica que Laudan no suministra»37. Pero es que en la ontología naturalista propiamente dicha no hay plataforma epistemológica posible 38 . Dentro del naturalismo biológico por el que habría siempre que empezar antes de ir a las vertientes de la psicología cognitiva y de la sociología del conocimiento (científico)- la

36 Para L. Laudan 1977, 1984, 1990 la ciencia -se refiere a la tecnología y, en todo caso, a la tecnociencia- mostraría una fiabilidad creciente, pero no considera los condicionantes bioecológicos aquí mencionados.

37 Rosenberg op. cit. p. 7.

38 Esto es algo que, en efecto, el naturalismo comparte con el relativismo epistemológico, véase Rosenberg, op. cit., p. 26. Incidentalmente, esta consideraciónentre otras apuntadas en el texto- neutraliza la famosa crítica de H. Putnam 1982, sobre la imposibilidad de naturalizar la actividad racional. 
mera pretensión de adquirir 'conocimiento verdadero', como se viene argüyendo, es completamente absurda. La referencia exclusiva es al conocimiento que facilite la supervivencia, incluyendo -en el hombre fundamentalmente- anticipaciones cognitivas que instrumentan esa supervivencia. Pero, de nuevo, el concepto de Verdad o Realidad no tiene sentido físico en el mundo de los seres vivos. Dicho concepto de Verdad o Realidad, empero, sí puede tener sentido biológico como 'creencia' que propicie la supervivencia directamente, o como consecuencia de otras intenciones. Por ejemplo, el sentido antropomórfico sería adaptativo en el ser humano - a pesar de su falta de encaje en su fenotipo ampliadoporque en cierto momento pudo propiciar su supervivencia: pensar que los animales actúan como nosotros facilitaría su localización y posterior captura ${ }^{39}$. Del mismo modo, como afirma por ejemplo Hull40, pensar que existe una realidad pura y dura, ajena a nosotros, estimularía la investigación científica para conocer esa supuesta realidad -donde habría que sobrevivir-, actividad que de otra manera, en principio, no parecería tener suficiente estímulo.

Con el naturalismo, como con el positivismo lógico, vuelve, en el mejor de los casos, a diluirse al mínimo la actividad filosófica; antes quedaba como base remanente la lógica matemática, hoy queda la 'lógica' biológica, porque tanto la 'lógica' psicológica, como la social, si no son fácilmente (metodológicamente) reducibles a la primera, lo que no pueden es ser incompatibles con ella.

En efecto, la preocupación filosófica desaparece al considerar la filosofía como una actividad cuyas preguntas perennes se pueden contestar en el marco de la ciencia. Como dice el naturalista Giere, la «gente mejor preparada para tratar sobre estas cuestiones [filosóficas] no son aquéllos formados como filósofos, sino aquéllos que están totalmente inmersos en la disciplina científica - o sea, los científicos» ${ }^{41}$. Marco que, por otra parte, puede tener una naturaleza tan metafísica como la puesta en escena tradicional, pero que implica una concepción distinta, como en su día ocurriera con el positivismo lógico que aunque rechazara la dimensión metafísica, ese mismo rechazo ya implicaba una postura al respecto.

Pero en el naturalismo, con énfasis biológico, la relación de la metafísica con las actitudes humanas sería análoga a la que existe en el 'medio exterior' con respecto al organismo que lo habita. El organismo

M. Douglas op. cit.

D. Hull op. cit.

4 R. Giere op. cit. p. xvi. 
mantiene constantemente una dinámica adaptativa con el medio. Y cuando cambia el medio cambia el proceso adaptativo que siempre va a remolque (las preadaptaciones son raras en el proceso evolutivo) ${ }^{42}$. Lo mismo sucede con la metafísica considerada como parte fundamental del medio humano. Se insiste que no tiene sentido biológico (¿puede haber otro sentido, desde el naturalismo?) asegurar que el clima metafísico pueda ser o bien real, o bien acercarse a la realidad, o bien ser ficticio. Las metafísicas vigentes serían los medios donde se desarrollan nuestras teorías y pretensiones varias, o sea serían los paradigmas kuhnianos en el sentido más general, pero, para repetir, sin los epítetos de verdadero, falso, casi falso, ficticio, poco verdadero, etc. El marco metafísico simplemente funcionará mejor o peor con vistas a nuestra supervivencia actual.

De manera que, por poner otro ejemplo, el concepto de similitud con la realidad (mapa isomórfico con la realidad hipotética) que defiende Giere -desde la perspectiva darwiniana ortodoxa- para justificar al menos un amago de imagen realista ${ }^{43}$, queda descartado acertadamente por Rosenberg («en el mejor de los casos, cualquier consideración darwiniana apoyará la operatividad de modelos sucesivos como un aumento de la capacidad de supervivencia local, y no en algún tipo de similitud absoluta con la realidad»44).

\section{CONCLUSIÓN}

Para que el naturalismo, como corriente 'epistémica', sea coherente hay que tener presente su base biológica. Esta base debe ser compatible tanto con las aproximaciones naturalistas desde la psicología cognitiva como desde la sociología del conocimiento. Pero dicha plataforma biológica impondría restricciones, al parecer insospechadas para la casi generalidad de los teóricos del naturalismo actual. Es decir, al ser -desde la perspectiva del naturalismo biológico-cualquier manifestación humana una característica orgánica más - relativamente adaptada y/o adaptativa, según los

42 Se hace hincapié que -desde la perspectiva biológica- lo que cambia es el fenotipo ampliado, y el proceso adaptativo no sería más que la búsqueda de una nueva coherencia estable entre las partes de dicho fenotipo ampliado variable.

43 Imagen, por otra parte, defendida por los epistemólogos evolucionistas y contemplada bajo la expresión campbeliana de 'realismo hipotético' (véase, K. Lorenz op. cit. -posiblemente la consideración más notable al respecto- junto con la oportuna crítica de M. Ruse 1987).

44 Rosenberg op. cit. p. 12. 
casos- cualquier declaración referente a que el proceso científico sea progresivo, tanto en su aspecto instrumentalista como, por supuesto, epistémico, carecería de sentido, es decir, incurriría en contradicciones insalvables. Otro tanto se puede proclamar, a) sobre la existencia de una supuesta realidad independiente, fuera ésta cognoscible (realismo) o no (antirrealismo) y b) sobre la pretensión de que "el hombre construye su realidad'. Ninguno de estos asertos tiene cabida en un naturalismo biológico en su raíz.

\section{REFERENCIAS BIBLIOGRÁFICAS}

ARNOLD, D. 1996: The Problem of Nature (Environment, culture and European expansion). Oxford: Blackwell.

AYER, A, 1971: The Problem of Knowledge. Middlesex: Penguin.

BERGER, P. L. y TH. LUCKMANN, 1966: The Social Construction of Reality (A treatise in the sociology of knowlege). New York: Doubleday.

CASTRODEZA, C. 1988: Ortodoxia Darwiniana y Progreso Biológico. Madrid: Alianza Universidad.

- 1997: «La evolución sin Darwin (la biología ultramontana)», Revista de Libros, 9, pp. $3-8$.

CHURCHLAND, P. M. 1984: Matter and Conciousness: A Contemporary Introduction to the Philosophy of Mind. Cambridge. MA: MIT Press.

CICOUREL, A. V. 1974: Language and Meaning in Social Interaction. New York: Free Press.

DAWKINS, R, 1976: The Selfish Gene. Oxford: Oxford University Press.

- 1980: The Extended Phenotype. Harlow: Longman.

DENNETT, D. C., 1995: Darwin's Dangerous Idea (Evolution and the meanings of life). New York: Simon \& Schuster.

DOUGLAS, M. 1990: «The pangolin revisited: a new approach to animal symbolism» en R. G. Willis (ed.), Signifying Animals: Human Meaning in the Natural World. London: Unwin Hyman, pp. 25-42.

GHISELIN, M. T. 1974: The Economy of Nature and the Evolution of Sex. Berkeley: University of California Press.

GIERE, R. 1988: Explaining Science: A cognitive approach. Chicago: Chicago University Press.

GOODWIN, B. 1995: How the Leopard Changed its Spots (The evolution of complexity). Londres: Phoenix.

GOULD, S. J. 1996: Full House (The spread of excellence from Plato to Darwin). New York: Harmony Books.

GOWER, B. 1997: Scientific Method (An historical and philosophical introduction). New York: Routledge.

HAMILTON, W. D. 1964: «The Genetical Evolution of Social Behaviour» (I y II), Journal of Theoretical Biology, VII, pp. 1-16 y 17-32.

HOOKER, C. A. 1996: Reason, Regulation and Realism (Toward a regulatory systems theory of reason and evolutionary epistemology). New York: SUNY Press.

HULL, D. 1988: Science as a Process: An evolutionary account of the social and conceptual development of science. Chicago: Chicago University Press. 
KAUFFMAN, S. 1995: At Home in the Universe (The search of laws of self-organization and complexity). London: Viking.

KIMURA, M. 1983: The Neutral Theory of Molecular Evolution. Cambridge: Cambridge University Press.

KITCHER, P. 1993: The Advancement of Science: Science without legend, objectivity without illusions. Oxford: Oxford University Press.

LAKOFF, G. 1995: «Embodied minds and meanings» en P. Baumgartner y S. Payr (eds.) Speaking Minds. Primaton: Princeton University Press, pp. 115-29.

LAUDAN, L. 1977: Progress and its Problems. Berkeley: University of California Press.

- 1984: Science and Values. Berkeley: University of California Press.

- 1990: Science and Relativism (Some key controversies in the philosophy of science). Chicago: The University of Chicago Press.

LORENZ, K. 1977: Die Rückseite des Spiegels (Versuch einer Naturgeschichte menschlichen Erkennens). München: DTV.

LOVEJOY, A. 1936: The Great Chain of Being (A study in the history of an idea). Cambridge MA: Harvard University Press.

NAGEL, T. 1974: «What is it like to be a bat?» Philosophical Review, LXXXIII, pp. 435-50.

PASSET, R. 1979: L'Economique et le Vivant. Paris: Payot.

PONTING, C. 1992: A Green History of the World. London: Penguin Books.

PUTNAM, H. 1982: «Why reason can't be naturalized», Synthèse, LII, pp. 3-23.

QUINE, W. V. O. 1951: From a Logical Point of View. Cambridge MA: Harvard University Press.

- 1969: Ontological Relativity. Nueva York: Columbia University Press.

RIDLEY, M. 1996: The Origins of Virtue. London: Viking.

ROSENBERG, A. 1996: «A field guide to recent species of naturalism», British Journal for the Philosophy of Science, XLVII, pp. 1-29.

RUSE, M. 1987: Tomándose a Darwin en Serio (Implicaciones filosóficas del darwinismo) tr. M. Vicedo. Barcelona: Salvat.

SKYRMS, B. 1996: Evolution of the Social Contract. Cambridge: Cambridge University Press.

STOKES, K. M. 1992: Man and the Biosphere (Toward a coevolutionary political economy). London: Sharpe.

SUTHERLAND, S. 1996: Irracionalidad (El enemigo interior), tr. C. González. Madrid: Alianza.

WILLIAMS, G. C. 1966: Adaptation and Natural Selection (A critique of some current evolutionary thought). Princeton: Princeton University Press.

WILSON, E. O. 1975: Sociobiology: The New Synthesis. Cambridge MA: The Belknap Press of Harvard University Press.

WOOLGAR, S. 1991: La Ciencia: Abriendo la Caja Negra, tr. E. Aibar. Barcelona: Anthropos. 